\title{
Performance Evaluation of SANQ System Identification as a Fault Diagnosis Method Under a Stochastic Situation
}

\author{
Haruyoshi Kamata, Kousuke Kumamaru, and Katsuhiro Inoue \\ Faculty of Computer Science and Systems Engineering, Kyushu Institute of Technology \\ Kawazu 680-4, Iizuka 820-8502, Fukuoka, Japan \\ e-mail:haruyosi@matsu.ces.kyutech.ac.jp
}

\begin{abstract}
We have constructed a failure diagnosis system using a quick identification method named as SANQ system identification [1], and verified the effectiveness and performance under the deterministic situation. However when the application of this method is considered, it is necessary to investigate the performance under the stochastic situation caused by, e.g. disturbances and measurement noises.

In this paper, we will verify characteristics and tolerance of the SANQ method under a stochastic situation, through simulation studies for a ship propulsion system. And we will try to modify the algorithm of the SANQ method so as to improve its performance under such a situation.
\end{abstract}

\section{Introduction}

It is important issue in maintenance engineering to construct a practical failure diagnosis system. There are various approaches for falul diagnosis [2], and identificationbased fault diagnosis method is one of them. The most of faults in dynamic systems can be modeled as unexpected fluctuation of system parameters. Therefore, such faults can be detected and isolated by monitoring of the parameters as fault factors.

We have constructed a failure diagnosis system using a quick identifcation method, which is named as "SANQ (Simulator based Approach to Neat and Quick)" system identification. This identification method can accommodate various types of nonlinear systems described in state-space form. We have applied this method to a ship governor model and a ship-propulsion system model, and verified the effectiveness and performance for the fault diagnosis under the deterministic situation.

However, when considering practical application of this method, it is nessesary to investigate the performance and characteristics under the stochastic situation. As the stochastic factors, disturbances in system dynamics and measurement noises in sensors are considered.

In this paper,we will verify the performace and characteristics of the diagnosis method under a stochastic situation caused by measurement noises, through simulation studies for a ship propulsion system [3]. And we will try to modify the algorithm of the SANQ method to improve its performance under such situation.

\section{SANQ System Identification Method}

In this section, we will outline the basic formula of the SANQ system identification method.

In this mehod, let us consider continuous-time nonlinear systems whose dynamics can be expressed by the state-space form as a physical model. Such systems are described by non-linear dynamic and measurement equations,

$$
\begin{aligned}
& \frac{d}{d t} x_{p}(t)=F\left(x_{p}(t), u_{p}(t), w(t), t ; \theta_{p}\right) \\
& z_{p}\left(t ; \theta_{p}\right)=M\left(x_{p}(t), u_{p}(t), v(t), t ; \theta_{p}\right)
\end{aligned}
$$

where, $x_{p}(t)$ is the state variable, $z_{p}\left(t ; \theta_{p}\right)$ the output variable measured by sensors, $u_{p}(t)$ the input siginal, $w(t)$ the disturbance imposed on plant dynamics. $v(t)$ is measurement noise, and $\theta_{p}$ denotes the plant parameter.

In the SANQ identification method, it is asumued that a simulator with the same structure as that of the plant dynamics can be prepared. Then the simulator is constructed by

$$
\begin{aligned}
& \frac{d}{d t} x_{s}(t)=F\left(x_{s}(t), u_{p}(t), t ; \theta_{s}\right) \\
& z_{s}\left(t ; \theta_{s}\right)=M\left(x_{s}(t), u_{p}(t), t ; \theta_{s}\right)
\end{aligned}
$$

where $x_{s}(t)$ is the simulator state variable, $z_{s}\left(t ; \theta_{s}\right)$ the output of the simlator, and $\theta=s$ denotes simulator parameter. Note that stochastic variables $w(t)$ and $v(t)$ are excluded from $F(\cdot)$ and $M(\cdot)$ in (1),(2). Thus the simulator dynamics are deterministic one.

In the SANQ method, the concept is based on an idea such that the simulator parameter is adjusted by successive perturbation procedure so as to minimize the output error between plant and simulator in the identification interval $\left[\begin{array}{ll}t_{0} & t_{0}+T\end{array}\right]$ (See Fig.1). The initial states 


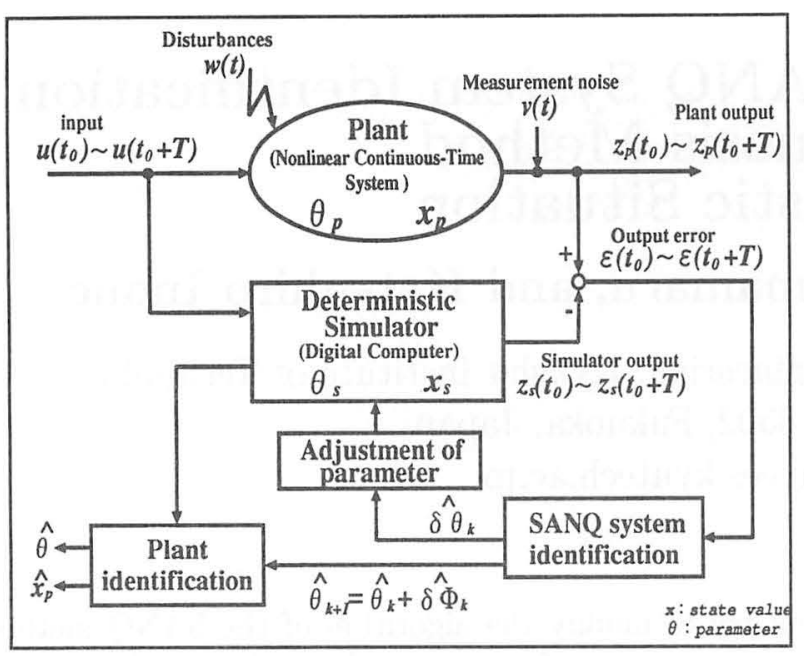

Fig.1 SANQ sytem identification

of simulator are included as adjustable parameters just like the simulator parameter vector to be adjusted and it consists of $\theta_{p}$ and $x_{s}\left(t_{0}\right)$. by,

The criterion function based on this concept is given

$$
J_{k}(\theta)=\int_{t_{0}}^{t_{0}+T}\left\|z_{p}\left(t ; \theta_{p}\right)-z_{s}\left(t ; \theta_{s}\right)\right\|_{R}^{2} d t
$$

$R$ is the weighting matrix which is appropriately selected by considering statistics of measurement noises, e.g. covariance matrix. So far, in the deterministic case, weighting matrix $\mathrm{R}$ have not been considered.

From the criterion function, we have the following estimation algorithm,

$$
\begin{aligned}
& \widehat{\delta}_{s}(k+1)= \\
& \left\{\int_{t 0}^{t 0+T} \frac{\partial z_{s}^{T}\left(t ; \hat{\theta}_{s}(k)\right)}{\partial \theta} \cdot R \cdot \frac{\partial z_{s}\left(t ; \hat{\theta}_{s}(k)\right)}{\partial \theta^{T}} d t\right\}^{-1} . \\
& \int_{t 0}^{t 0+T} \frac{\partial z_{s}^{T}\left(t ; \hat{\theta}_{s}(k)\right)^{T}}{\partial \theta} \cdot R \cdot\left\{z_{p}\left(t ; \theta_{p}\right)-z_{s}\left(t ; \hat{\theta}_{s}(k)\right)\right\} d t
\end{aligned}
$$

$$
\begin{array}{r}
\hat{\theta}_{s}(k+1)=\hat{\theta}_{s}(k)+\widehat{\delta \theta}_{s}(k) \\
k=1,2, \cdots
\end{array}
$$

where, $\partial z_{s}\left(t ; \hat{\theta}_{s}(k) / \partial \theta_{s}\right.$ in (6) is a sort of parameter sensitivity of the simulator output, and subjects to a differential equation derived from the simulator dynamics (3),(4). Thus, we can estimate the plant parameter $\theta_{p}$ together with the plant initial state $x_{p}\left(t_{0}\right)$ from adjusted simulator parameter $\hat{\theta}_{s}(k)$, where $k$ denotes iteration number.

The criterion (5) seems to be a standard one for the output error type of identification. However, the identification interval $T$ can be taken to be a short interval comparable with the duration time of transient response, because the initial states $x_{s}\left(t_{0}\right)$ are included as adjustable parameters in this method. Therefore, the SANQ method makes it possible to execute a quick identification by using short time record of input-output data.

In this method, the identifiability is equivalent to existence of the inverse matrix in (6), that means independentness of $\frac{\partial}{\partial \theta} z_{s}\left(t ; \theta_{s}\right)$ over the identification interval $\left[\begin{array}{ll}t_{0} & t_{0}+T\end{array}\right]$.

\section{Decentralized Failure Diagnosis System}

As is mentioned, the SANQ method contains state variables as adjustable parameters. Therefore, for plant with high dimension, the number of parameters to be estimated inevitably increases. Due to the fact, there may be considered the case that the identifiability isn't satisfied, or cost of calculation increases. To solve these problems, we construct "A Decentralized Failure Diagnosis System" as shown in Fig2.

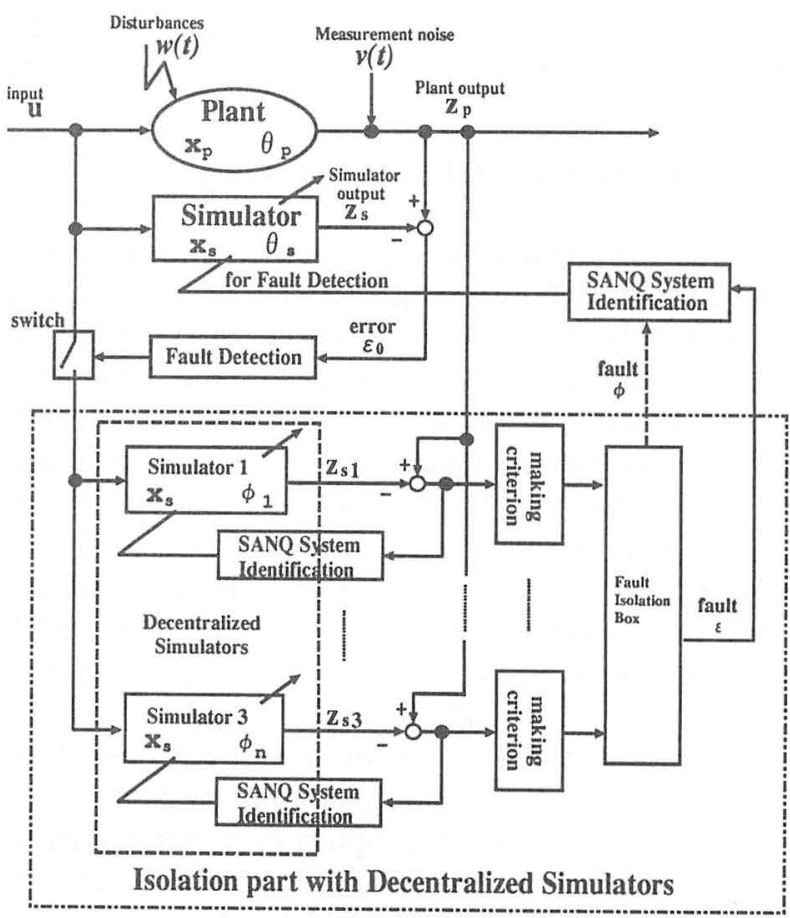

Fig.2 Decentralized failure diagnosis system

The procedure of a decentralized failure diagnosis system is as follows:

First, a model with the same structure as that of plant dynamics is prepared for a simulator, and a fault is detected by comparing the outputs of the plant and the simulator. When a fault caused by unexpected change 
of plant parameters has occurred, the effect will appear in the plant output. Therefore, it is possible to detect a fault from the output error between the plant and the simulator.

Then, after the fault detetion, input and output data generated from the plant and the simulator are delivered to each decentralized simulator. Parameters which may be fault factor are estimated by using SANQ system identification method in each decentralized simulator.

Next, the fault factor is decided from the output error between the plant and each decentralized simulator as the result of estimation. Finally, in order to evaluate the fault degree, paramter set is estimated based on the identification results in decentralized simulators.

\section{Ship-Propulsion System}

In this section, as the plant to be diagnosed, we will consider a ship-propulsion system, which has one engine and one propeller. This system-model has been proposed for benchmark test of fault tolerant control by IzadiZamanabadi,M.Blanke et al, and is the model for a low speed marin vehicle.

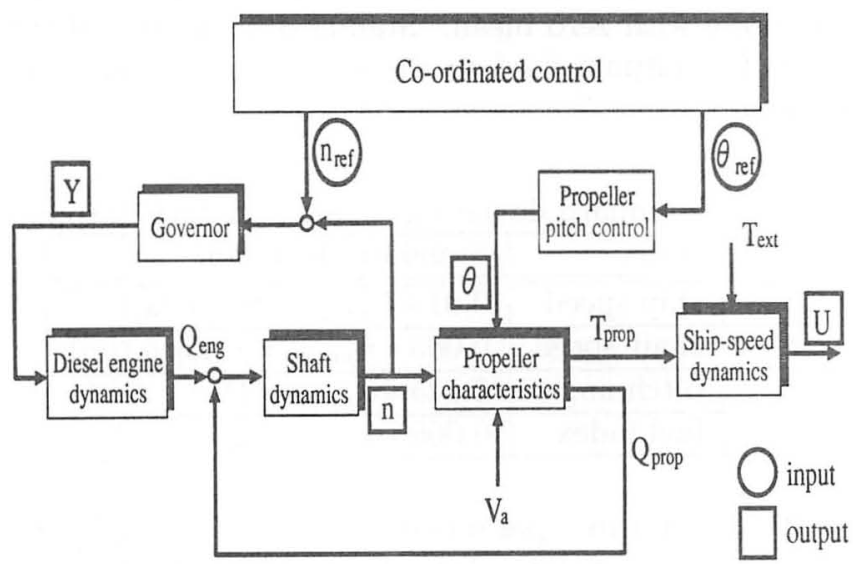

Fig.3 Schematic diagram of the ship speed propulsion system

\subsection{An overview of the ship-propulsion system}

This system has following several features.

- The model of a small container ship. (length:147.2m,displacement: $12,840 \mathrm{~m}^{3}$ )

- Various non-linearities are faithfully modeled.

- Six ordered non-linear system which has two inputs and four outputs.

- Various stochastic factors are modeled. (measurement noise, external force etc...)
The schematic diagram of this system is shown in Fig.3. Main components/subsystems of the system are:

- Propeller pitch control system.

- Governor.

- Diesel engine.

- Propeller characteristics.

- Ship speed dynamics.

This system has two inputs and four measurable outputs (See Fig.3). Inputs and outputs are following

- inputs

- the shaft speed reference: $n_{r e f}$

- the propeller pitch reference: $\theta_{r \varepsilon f}$

- outputs

- the diesel engine shaft speed: $n_{m}$

- propeller pitch position: $\theta_{m}$

- the fuel index: $Y$

- the ship speed: $U_{m}$

\subsection{Structure of the non-linear plant model}

Each subsytems is thoroughly described in the following.

\section{- Propeller pitch control system}

The propeller pitch system is approximated by a simple integrator, and the system is controled by a P-controller, and is described by following equations:

$$
\begin{aligned}
& \theta_{m}=\theta+\nu_{\theta}+\Delta \theta \\
& u_{\dot{\theta}}=k_{t}\left(\theta_{\text {ref }}-\theta_{m}\right) \\
& \dot{\theta}=\max \left(\dot{\theta}_{\min }, \min \left(u_{\dot{\theta}}, \dot{\theta}_{\max }\right)\right)+\Delta \dot{\theta}_{i n c} \\
& \theta=\max \left(\theta_{\min }, \min \left(\theta, \theta_{\max }\right)\right)
\end{aligned}
$$

where $\theta_{m}$ is the measured propeller pitch, $\left(\dot{\theta}_{\max }, \dot{\theta}_{\min }\right)$ are the limits set by the hydraulic pump,and $\left(\theta_{\max }, \theta_{\min }\right)$ are the physical limits for propeller. $\nu_{\theta}$ is the measurement noise. The available signals are $\theta$ and $\theta_{\text {ref }}$.

\section{- Governor}

Governor mainly consists of a PI-controller. Input to the Governor is the difference between shaft speed reference $n_{r e f}$ and measured shaft speed $n_{m}$. The output is the fuel Index $Y$. An anti-windup arrangement is included in the Governor.

$$
\begin{aligned}
& n_{m}=n+\nu_{n}+\Delta n \\
& \dot{Y}_{i}=\frac{k_{r}}{\tau_{i}}\left(\left(n_{r e f}-n_{m}\right)-K\left(Y_{P I}-Y_{P I b}\right)\right) \\
& Y_{P I b}=Y_{i}+k_{r}\left(\left(n_{r e f}-n_{m}\right)\right) \\
& \left.Y_{P I}=\min \left(\max \left(Y_{P I b}, Y_{l b}\right), Y_{u b}\right)\right)
\end{aligned}
$$


Due to physical constraints, the fuel index generated by governor is modified by,

$$
y_{\max }= \begin{cases}1 & \text { if } n_{m} \geq 0.8 n_{\max , a}, \\ \frac{1.5}{n_{\max , a}} n_{m}-0.2 & \text { if } 0.4 n_{\max , a}<n_{m}<0.8 n_{\max , a}, \\ 0.4 & \text { if } n_{m} \leq 0.4 n_{\max , a}\end{cases}
$$

$$
Y=\max \left(0, \min \left(Y_{P I}, y_{\max }\right)\right)
$$

\section{- Diesel Engine Dynamics}

According to the fuel index, the diesel engine generate a torque, $Q_{\text {eng }}$. The relation between generated torque and fuel index is described by following equation:

$$
\frac{Q_{\text {eng }}}{Y}(s)=\frac{\left(k_{y}+\Delta k_{y}\right) e^{-\tau s}}{1+\tau_{c} s}
$$

Then the torque turns the shaft. When considering the applied torques to the shaft, following differential equation is given:

$$
I_{m} \dot{n}=Q_{e n g}-Q_{\text {prop }}-Q_{f}
$$

$Q_{e n g}[\mathrm{Nm}]$ is the torque developed by diesel engine, $Q_{\text {prop }}[\mathrm{Nm}]$ is the developed torque from propeller $\mathrm{dy}-$ namics, and $Q_{f}[\mathrm{Nm}]$ is the friction torque.

\section{- Propeller Characteristics}

The propeller pitch is the angle between a single propeller blade and its turning direction. The developed propeller thrust and propeller torque are determined by following bilinear relation:

$$
\begin{array}{r}
T_{\text {prop }}=T_{|n| n}(\theta)|n| n+T_{|n| V a}(\theta)|n| V_{a} \\
Q_{\text {prop }}=Q_{0|n| n}+Q_{|n| n}(\theta)|n| n+Q_{|n| V_{a}}(\theta)|n| V_{a}
\end{array}
$$

where $V_{a}[\mathrm{~m} / \mathrm{s}]$ is the velocity of the water that goes into the propeller disc and $n[\mathrm{rad} / \mathrm{s}]$ is the shaft speed. $Q_{0}$ denotes the torque which is produced by the propeller when the pitch is zero.

The coefficients $T_{|n| n}, T_{|n| V a}, Q_{|n| n}, Q_{|n| V_{a}}$ are complex functions of the pitch angle $\theta$.

\section{- Ship Speed Dynamics}

The ship speed dynamics is described by:

$$
\begin{array}{r}
\left(m-X_{\dot{U}}\right) \dot{U}=R(U)+(1-t) T_{\text {prop }}-T_{\text {ext }} \\
U_{m}=U+\nu_{U}
\end{array}
$$

$R(U)$ describes the resistance of the ship in the water and is negative quantity. $X_{\dot{U}}$ represents the added mass in surge and is a negative quantity. $T_{e x t}$ represents the external force imposed on the ship motion by wind and waves. $U_{m}$ is the measured ship velocity and $\nu_{m}$ is the measurement noise.

The following variables are included as stochastic factors in this system.
- measurement noise: $\nu_{\theta}, \nu_{n}, \nu_{U}, \nu_{Y}$

- the velocity of the water which goes into the propeller disc: $V_{a}$

- the external force imposed on the ship motion: $T_{e x t}$

\section{Simulation Studies}

\section{under a Stochastic Situation}

Simulation studies on paramter identification and fault diagnosis of the ship propulsion system have been carried out.

As is mentioned, so far, the effectiveness and performance of the SANQ method for fault diagnosis have been verified under the deterministic situation. In this section, the performance of this method is verified under a stochastic situation only caused by measurement noises. On the other hand, the stochastic factors in dynamics equation are left to be future studies.

\section{Specifications}

Each output is measured with additive noise. The noise is assumued to be a normal distributed white Gaussian noise with zero mean. Standard deviations of the noises for outputs used in the simulation are given in Table 1.

Table 1: standard deviation of noise for each output

\begin{tabular}{|c|l|l|}
\hline Signal & Name & Standard deviation \\
\hline \hline$U$ & ship speed & $0.01 * U_{\max }(=9.7[\mathrm{~m} / \mathrm{s}])$ \\
\hline$n$ & shaft speed & $0.005 * n_{\max }(=12.5[\mathrm{rad} / \mathrm{s}])$ \\
\hline$\theta$ & pitch angle & $0.425 * \theta_{\max }(=1)$ \\
\hline$Y$ & fuel index & $0.005 * Y_{\max }(=1)$ \\
\hline
\end{tabular}

We select three parameters as fault factors in the ship-propulsion system (Table 2), and identify them using the SANQ method when each parameter change has occurred individually.

Table 2: parameters as fault factor

\begin{tabular}{|l||l|l|l|}
\hline par. & description & value & unit \\
\hline \hline$k_{y}$ & engine gain & $1.137 \times 10^{6}$ & \\
\hline$I_{m}$ & inertia & $0.25 \times 10^{6}$ & $\mathrm{kgm}^{2}$ \\
\hline$k_{t}$ & pitch angle control gain & 0.15 & \\
\hline
\end{tabular}

Further, assuming the independence of each noise, we will select weighting matrix $\mathrm{R}$ to be diagonal matrix. Its factors are variances of measurement noises in outputs,

$$
R=\left[\begin{array}{cccc}
\operatorname{cov}\left(\nu_{n}\right) & 0 & 0 & 0 \\
0 & \operatorname{cov}\left(\nu_{\theta}\right) & 0 & 0 \\
0 & 0 & \operatorname{cov}\left(\nu_{U}\right) & 0 \\
0 & 0 & 0 & \operatorname{cov}\left(\nu_{Y}\right)
\end{array}\right]^{-1}
$$

The fault modes are specified by following three cases: 
1. The change of $k_{y}$.

2. The change of $I_{m}$.

3. The change of $k_{t}$.

The other specifications are considered as follows and they are explained in Fig.4.

- data interval for simulation: $[0 \sim 3500] \mathrm{sec}$

- the time when the parameter was changed: at $500 \mathrm{sec}$

- the degree of paramter change: $+10 \%$

- identification interval: $T=60[\mathrm{step}](=60 \mathrm{sec})$

- iteration number of the SANQ method: 20times

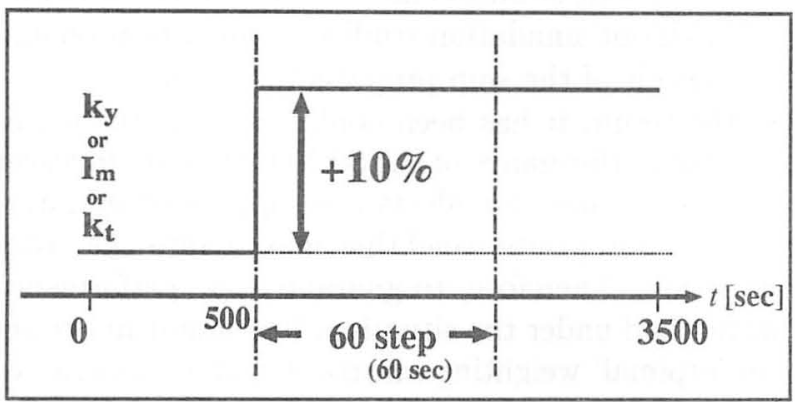

Fig.4 Specification of simulation studies

\subsection{Simulation results}

The simulation results are shown in Fig. 5 for the case 1 , in Fig.6 for the case 2, in Fig.7 for the case 3, while Fig.8 $\sim$ Fig.10 show the results without a weighting matrix $R$ in the SANQ method. In each case, estimated values of plant parameters as the fault factors and their true values are plotted.

The estimation errors are evaluated by normalized values in Table 3 and Table 4, where $\epsilon_{1}$ denotes estimation errors with weighting matrix $R$, and $\epsilon_{2}$ denotes those without $R$.

Table 3: estimation error for initial estimates

\begin{tabular}{|l||l|l|l|}
\hline & case 1(ky) & case 2(Im) & case 3(kt) \\
\hline$\epsilon_{1}$ & 0.08061 & 0.02530 & 0.09234 \\
\hline$\epsilon_{2}$ & 0.13602 & 0.17623 & 0.13429 \\
\hline
\end{tabular}

Table 4: estimation error for parameters

\begin{tabular}{|l||l|l|l|}
\hline & case 1(ky) & case 2(Im) & case 3(kt) \\
\hline$\epsilon_{1}$ & 0.00118 & 0.00062 & 0.00637 \\
\hline$\epsilon_{2}$ & 0.00243 & 0.00455 & 0.00188 \\
\hline
\end{tabular}

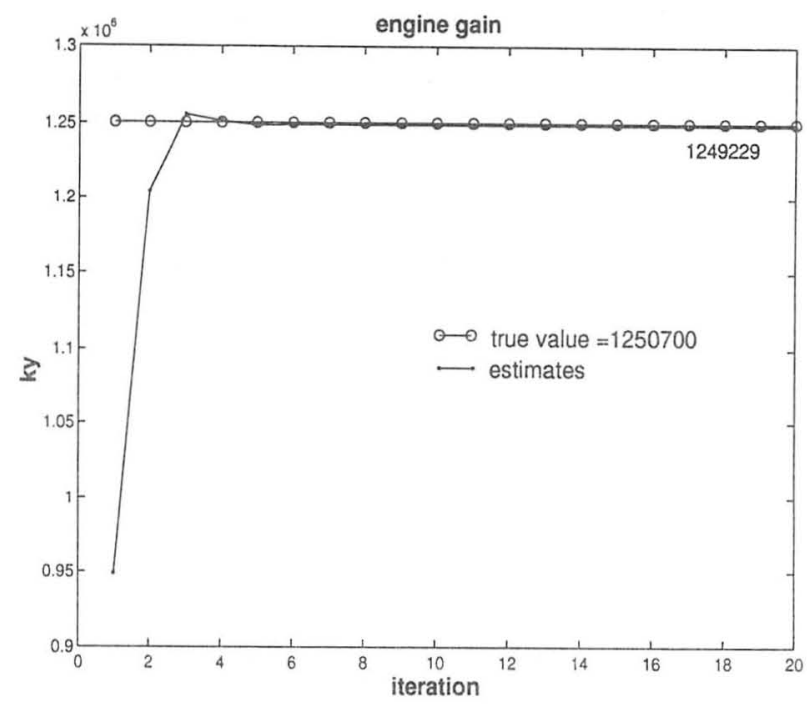

Fig.5 Simulation result (weighted) case 1: change of $k_{y}$

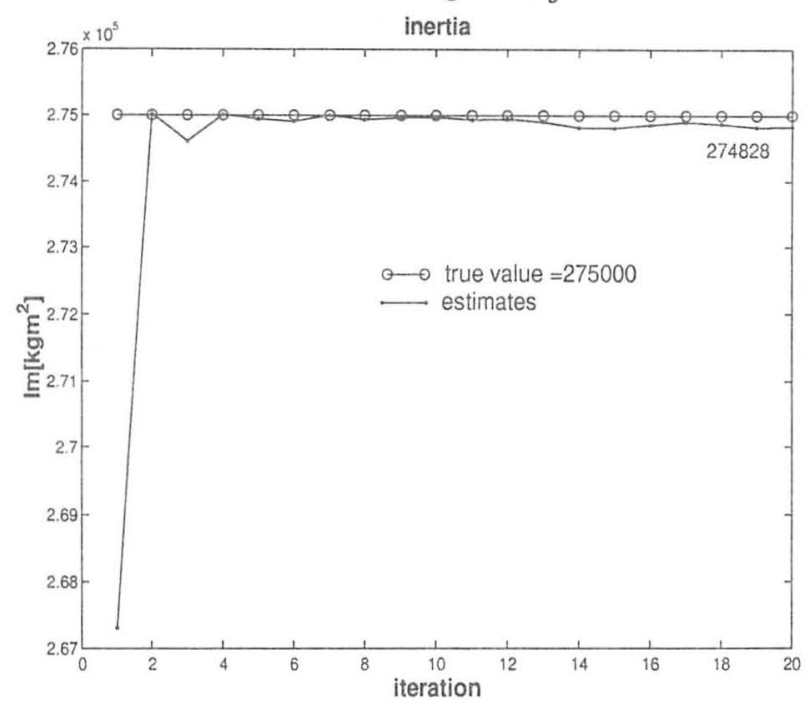

Fig.6 Simulation result (weighted) case 2: change of $I_{m}$ pitch control gain

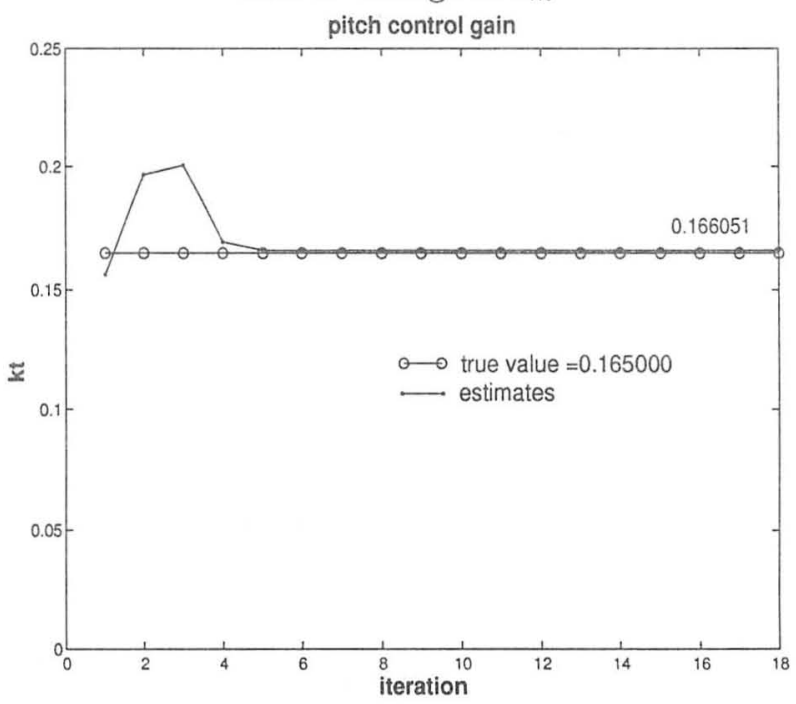

Fig.7 Simulation result (weighted) case 3: change of $k_{t}$ 


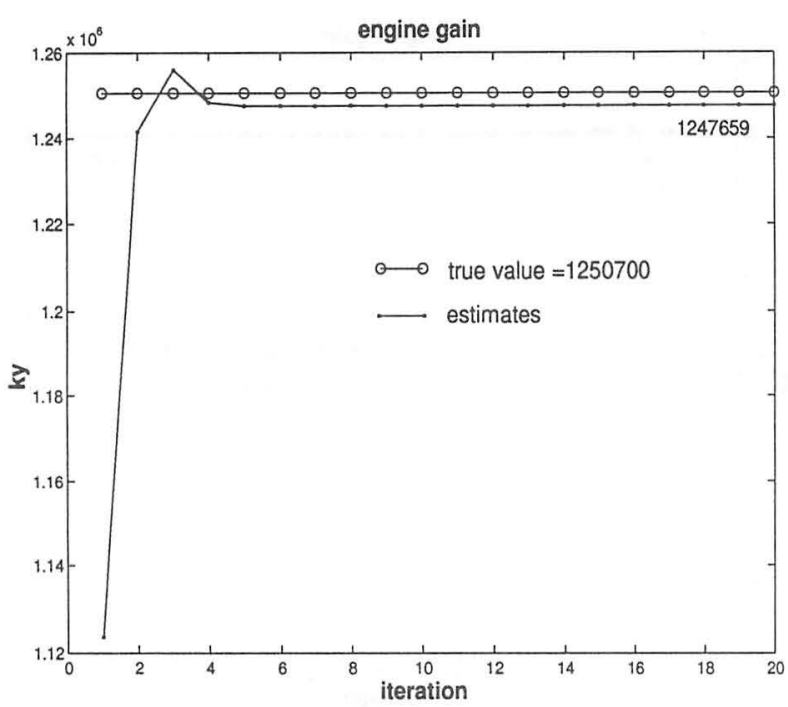

Fig.8 Simulation result (no-weighted) case 1: change of $k_{y}$.

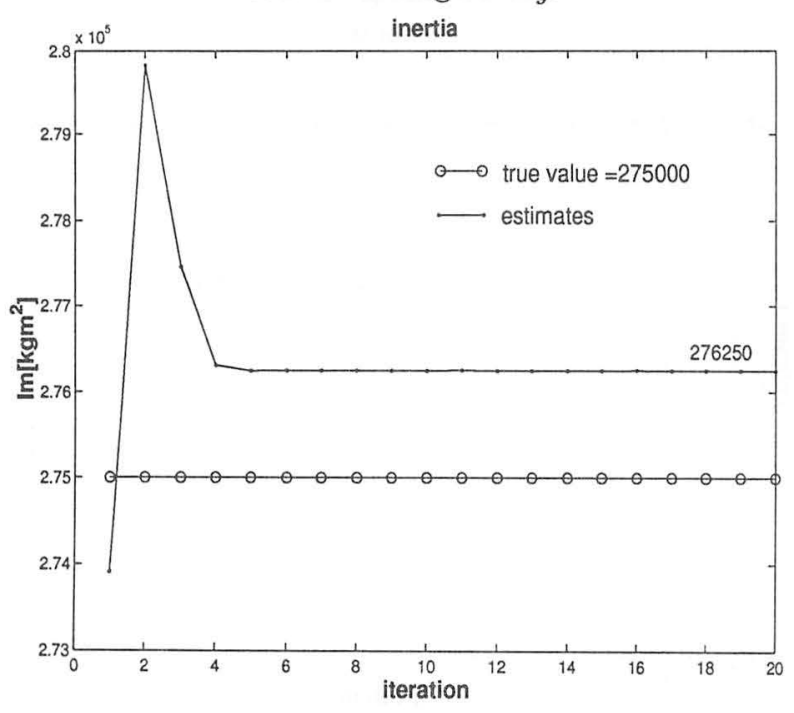

Fig.9 Simulation result (no-weighted) case 2: change of $I_{m}$

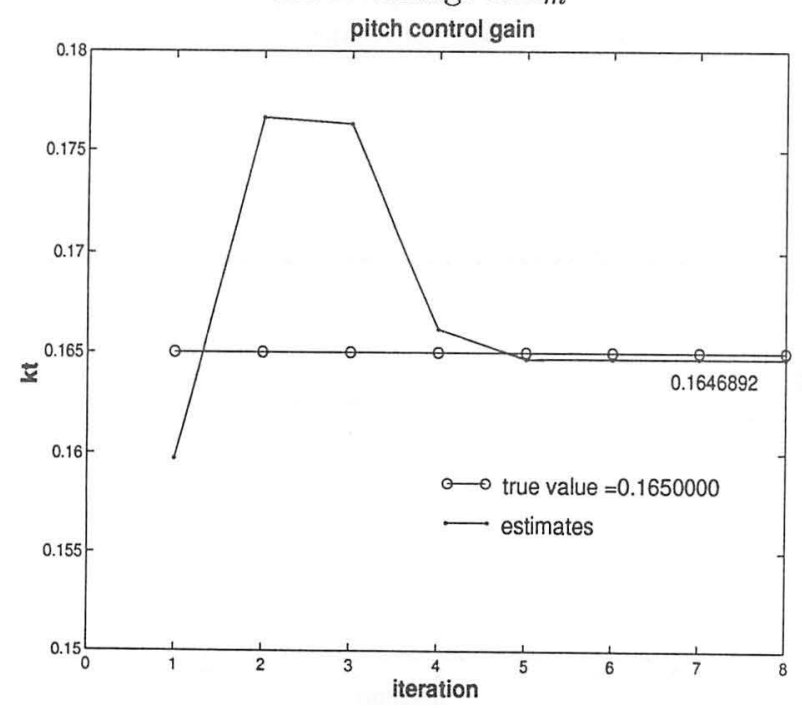

Fig.10 Simulation result (no-weighted) case 3: change of $k_{t}$

\subsection{Discussions}

From Fig.8 Fig.10, we can see that some degree of bias is appeared in each estimated result in the case without weighting matrix $R$. On the other hand, in the case with weighting matrix, it can be seen that the effect of bias is suppresesd (See Fig.5 Fig.6).

It can be seen from Table 3 and Table 4 that a reasonable estimation accuracy is obtained.

Therefore, when a system has a stochastic factor like a measurement noise, appropriate weighting matrix can be one of solutions to suppress its effects.

\section{Conclusions}

In order to examine the performace of the SANQ method under a stochastic situation like a measurement noise, we have carried out simulation studies on identification and fault diagnosis of the ship propulsion system.

As the result, it has been confirmed that the noises degrade the performance of the SANQ method. However it can be seen that the effects are suppressed by introducing an appropriate weighting matrix into the criterion function. Therefore, to guarantee the performance of this method under the situation, it is important to select an 'otpimal' weighting matrix. On this concern, we need more case studies, and it is important to develop another approach to this problem, too. The verification for other stochastic factors is still open problem to be investigated.

\section{References}

[1] M.Uchida et al: A Simulator-Based Quick Identification Method of Nonlinear Systems, Trans. of Society of Instrument and Control Engineers, Vol.30, No.8, p908-916 (1994)

[2] R.Isermann, P.Balle: Trends in The Application of Model Based Fault Detection and Diagnosis of Technical Processes, 13th World Congress of IFAC, volume N:p.1-12(1996)

[3] Roozbeh Izadi-Zamanabadi,Mogens Blanke : A Ship Propulsion System Model for Faulttolerant Control, Department of Control Engineering,Aalborg Univercity, Fredrik Bajers Vej7,Dk-9220 Aalborg, Denmark(1998) 